\title{
Large squares and sets of analyticity in tensor algebras
}

by

D. L. SALINGER (Orsay)

1. Introduction. If $X$ and $Y$ are compact Hausdorff spaces, $\nabla(X \times Y)$ $=\boldsymbol{C}(X) \hat{\otimes} \boldsymbol{C}(\bar{Y})$ will denote the projective tensor product of the Banach algebras $\boldsymbol{C}(X)$ and $\boldsymbol{C}(Y) . \nabla(X \times Y)$ is a regular symmetric Banach algebra under the projective norm: for a detailed discussion of its definitions and elementary properties see [11]; a summary of them is to be found in [10].

If $E$ is a closed subset of $X \times Y$, we define the closed ideal $I(E)$ by

$$
I(E)=\{f \in V(X \times Y): f(z)=0 \text { when } z \epsilon E\} .
$$

$V(E)=V(X \times Y) / I(E)$ is the algebra of restrictions to $E$ of functions of $V(X \times \bar{Y})$. If $V(E) \cong C(E)$, we call $E$ a $V$-Helson set.

Let $\varphi$ be a continuous complex-valued function defined on the interval $[-1,1]$ of the real line. $\varphi$ is said to operate on the algebra $V(E)$ if $\varphi \circ f \epsilon V(E)$ whenever $f \in V(E)$ has range in $[-1,1] . E$ is called a set of analyticity (for the algebra $V(X \times Y)$ ) if any function operating on $V(E)$ can be extended to an analytic function in a neighbourhood of $[-1,1]$; i.e. $E$ is a set of analyticity if "only the analytic functions" operate on $V(E)$.

For a compact abelian group $G$, we define $A(G)$ to be, as usual, the algebra of Gelfand (Fourier) transforms of $L^{1}(\hat{G})$, where $\hat{G}$ is the dual group of $G$. If $E$ is a compact subset of $G, I(E)$ and $A(E)$ are defined similarly to the respective cases above. If $A(E) \cong C(E), E$ is called a Helson set. $E$ is a set of analyticity. (for the algebra $A(G)$ ) if only the analytic functions operate on $A(E)$.

The dichotomy conjecture (cf. [t], [6]) is that every compact subset of a compact abelian group which is not a Helson set should be a set of analyticity. We can pose the same question in the context of the tensor algebra $V(X \times Y)$ and there too the answer is not known, however the following combinatorial characterization of countable $V$-Helson sets following combinatorial sufficient conditions for sets to be sets of analyticity. suggests combinatorial sufficient conditions for sets to be sets of analyticity.
If $X$ and $Y$ are compact Hausdorff spaces and $n$ is a positive integer, we call $n$-squares those subsets of $X \times Y$ of the form $S_{n}=X_{n} \times Y_{n}$, where $X_{n} \subset X, Y_{n} \subset Y$ and $\left|X_{n}\right|=\left|Y_{n}\right|=n$. 
THeOREM 1.1 (Varopoulos [11], 6.4]). A countable closed subset $E \subset X \times Y$ is a $V$-Helson set if and only if there exists a positive integer $\lambda$ such that $\left|E \cap S_{n}\right|<\lambda n$ for all $n$-squares $S_{n}$ and for all positive integers $n$.

In this paper, we consider the case of the intersection of a sequence of $n$-squares $\left\{S_{n}\right\}_{n=1}^{\infty}$ with $E \subset X \times Y$, showing first that if $\left|E \cap S_{n}\right|=n^{2}$, then $E$ is a set of analyticity. Then, by a combinatorial refinement we show that it is sufficient to have $\left|E \cap S_{n}\right| \geqslant n^{2-\varepsilon_{n}}$, where $\varepsilon_{n} \rightarrow 0$ as $n \rightarrow \infty$. For the remaining results we use the methods developed by Katznelson and Malliavin $[5,6]$ to show that if $n\left|E \cap S_{n}\right|^{-1} \rightarrow 0$, then $E$ is almost surely (with respect to a certain probability space) a set of analyticity. We also give similar results for a more specialised class of sets.

In conclusion, I would like to thank Dr. N. Th. Varopoulos for his extremely stimulating help and guidance. I want also to thank the S. R. C. and N.A.T. O. for their invaluable financial support.

2. LEMTIA 2.1. Let $X, Y$ be compact Hausdorff spaces and let $E$ be a closed subset of $X \times Y$. Let $E$ contain an $n$-square for every positive integer $n$.

Then $E$ is a set of analyticity for the tensor algebra $V(X \times Y)$.

Proof. By $\boldsymbol{T}$ we shall denote the group of complex numbers with unit modulus, and by $\boldsymbol{Z}(n)$ the cyclic group of order $n$. We shall identify $\boldsymbol{Z}(n)$ with its embedding in $\boldsymbol{T}$ as the group of $n^{\text {th }}$ roots of unity.

It is known [2] that there exists a constant $\alpha>0$ such that for any positive real number $R>0$ there exists a real function $f \in A(\boldsymbol{T})$ with

$$
\|f\|_{A(\boldsymbol{T})}<R \text { and }\left\|e^{i f}\right\|_{\boldsymbol{A}(\boldsymbol{T})}>e^{\alpha R} \text {. }
$$

Let $\varphi \in A^{*}(\boldsymbol{T})$ be such that $\|\varphi\|_{A^{*}}=1$ and $\left|\left\langle e^{i f}, \varphi\right\rangle\right|>e^{\alpha R}$. By the method of Herz [i], there exists a sequence $\left(\varphi_{n}\right)_{n=1}^{\infty}$ of pseudomeasures , $\varphi_{n} \in A^{*}(\boldsymbol{Z}(n))$ such that $\left\|\varphi_{n}\right\| \leqslant 1$ and $\varphi_{n} \rightarrow \varphi$ in the weak star topology. Hence there exists $n_{0}$ such that for all $n>n_{0},\left|\left\langle e^{i f}, \varphi_{n}\right\rangle\right|>e^{\alpha R}$ and thus $\|\left. e^{i f}\right|_{\boldsymbol{Z}_{(n)} \|_{A\left(\boldsymbol{Z}_{(n))}\right.}>e^{a R} \text {. So, fixing } n>n_{0} \text {, we have a real function }}$ $f_{1}=\left.f\right|_{\mathbf{Z}(n)} \dot{\epsilon} A(\boldsymbol{Z}(n))$ such that

$$
\left\|f_{1}\right\|_{A}<R \quad \text { and } \quad\left\|e^{i f_{1}}\right\|_{A}>e^{\alpha R} \text {. }
$$

Now let $S_{n}=X_{n} \times Y_{n}$ be an $n$-square in $E$. To transfer $f_{1}$ from $A(\boldsymbol{Z}(n))$ to $\boldsymbol{V}\left(S_{n}\right)$, we identify $S_{n}$ with the set $\boldsymbol{Z}(n) \times \boldsymbol{Z}(n)$ and we consider the map $M: A(\boldsymbol{Z}(n)) \rightarrow V\left(S_{n}\right)$ defined by

$$
M h(x, y)=h(x+y)
$$

for any $h \in A(\boldsymbol{Z}(n))$ and $x, y \in \boldsymbol{Z}(n)$. This map is an isometric isomorphism sidentifying $A(\boldsymbol{Z}(n))$ with a closed subalgebra of $V\left(S_{n}\right)$ ([11],8.1), 0 we have a real function $g_{1} \in V\left(S_{n}\right)$ satisfying

$$
\left\|g_{1}\right\|_{\nabla\left(S_{n}\right)}<R \quad \text { and } \quad\left\|e^{i g_{1}}\right\|_{V\left(S_{n}\right)}>e^{\alpha R}
$$

Hence there is a function $g \in V(E)$ taking only real values and having

$$
\|g\|_{\nu(E)}<2 R \text { and }\left\|e^{i g}\right\|_{\nu(E)}>e^{\alpha R}
$$

which is enough to show that $E$ is a set of analyticity [3].

Comparing this result with theorem 1.1, it is natural to ask whether we can glean information from the type of intersection of a sequence of $n$-squares with a particular set.

We shall say that a sequence $\left\{S_{n}\right\}_{n=1}^{\infty}$ of $n$-squares has incidence $d(n)$ in a subset $E$ of $X \times Y$ if $d(n)$ is a function on the positive integers such that

$$
\left|\left(E \cap S_{n}\right)\right| \geqslant d(n)
$$

for an infinity of values of $n$.

THEOREM 2.2. Let $X, Y$ be compact Hausdorff spaces, let $E$ be a closed subset of $X \times Y$ and let $\left\{S_{n}\right\}_{n=1}^{\infty}$ be a sequence of $n$-squares in $X \times Y$ having incidence $n^{2-\varepsilon_{n}}$ in $E$, where $\varepsilon_{n} \rightarrow 0$ as $n \rightarrow 0$.

Then $E$ is a set of analyticity for the tensor algebra $\nabla(X \times Y)$.

This theorem immediately follows from lemma 2.1 and the following proposition, due to Kővari, Sós and Turàn [8]:

Proposition 2.3. Let $X, Y$ be arbitrary sets, let $E$ be a subset of $X \times Y$ and let $\left\{S_{n}\right\}_{n=1}^{\infty}$ be a sequence of $n$-squares having incidence $n^{2-\varepsilon_{n}}$ in $E$, where $\varepsilon_{n} \rightarrow 0$ as $n \rightarrow \infty$.

Then we can find a sequence of $n$-squares $\left\{T_{n}\right\}_{n=1}^{\infty}$ such that $T_{n} \subset E$ for each $n$.

Proof. Without loss of generality we shall assume that $n^{\varepsilon} n$ is an integer. To avoid unnecessary complication we shall suppose also that $\left|\left(S_{n} \cap E\right)\right| \geqslant n^{2-\varepsilon_{n}}$ for every $n \geqslant 1$.

By rows (respectively columns) of $S_{n}$ we shall understand sets of the form $x_{0} \times Y_{n}$ (respectively $\left.X_{n} \times y_{0}\right)$, where $x_{0} \in X_{n}\left(y_{0} \in Y_{n}\right)$. Let $r$ be a positive integer and suppose $n_{0}$ is such that $\varepsilon_{n}<1 / r$ for $n \geqslant n_{0}$. For some such $n$ we select a subset $S_{n}^{\prime} \subset S_{n}$ consisting of $t=r n^{\varepsilon_{n}}$ rows of $S_{n}$ and containing at least $r n$ points of $E$. Let $s$ be the number of columns of $S_{n}^{\prime}$ containing at least $r$ points of $E$. Then, calculating an upper bound for the number of points in $S_{n}^{\prime}$, we obtain $s t+(n-s)(r-1) \geqslant r n$ and hence $s t \geqslant n$ for $r>1$ (the case $r=1$ is, of course, trivial). In any column of $S_{n}^{\prime}$ containing at least $r$ points of $E, r$ points of $E$ can be arranged in $\left(\begin{array}{l}t \\ r\end{array}\right)$ ways, so if $s>r\left(\begin{array}{l}t \\ r\end{array}\right)$, we shall have obtained an $r$-square $T_{r} \subset E$. To do this we observe that

$$
r\left(\begin{array}{l}
t \\
r
\end{array}\right)<t^{r}=r^{r} n^{r \varepsilon_{n}} \quad \text { and } \quad s \geqslant\left(n^{1-\varepsilon_{n}}\right) / r
$$

so if $n$ is large enough, we obtain the desired result. 
3. We follow Katznelson and Malliavin [5] in introducing the definitions below.

Let $F$ be a finite-dimensional vector space over $R$ and let \|\|$_{1},\|\cdot\|_{2}$ be two norms on $F$ such that \|\|$_{2} \leqslant\|\|_{1}$. Let \|\|$_{1}^{*}$ be the dual norm on the dual $F^{*}$ of $\left(F,\|\|_{1}\right)$. A set of majoration of \|\|$_{1}$ with respect to \|\|$_{2}$ is a subset $S$ of the unit ball of $\left(F,\|\|_{2}\right)$ such that

$$
\|\mu\|_{1}^{*} \leqslant 2 \sup _{S}|\langle\mu, f\rangle|
$$

for all $\mu \in F^{*}$. The scale of \|\|$_{1}$ compared with \|\|$_{2}$ is the cardinal of a smallest possible set of majoration. If $X, Y$ are two compact spaces and $E$ is a finite subset of $X \times Y$, the arithmetic diameter $d(E)$ of $E$ is the scale of \|\|$_{V(E)}$ compared with \|\|$_{C(E)}$ (this latter definition being applicable also when considering $\nabla(E), C(E)$ as complex vector spaces).

LEMMA 3.1. Let $X, Y$ be finite sets having $|X|=|Y|=n$, a positive integer. Then $d(E) \leqslant 2^{4 n}$ for any subset $E$ of $X \times Y$.

Proof. Let $\mu$ be a measure on $E$. Then

$$
\|\mu\|_{A^{*}(E)}=\sup |\langle\mu, f \otimes g\rangle|
$$

where $f_{\epsilon} \boldsymbol{C}(\bar{X}), g_{\epsilon} \boldsymbol{C}(\bar{Y})$ and $\|f\|_{\infty}=\|g\|_{\infty}=1$.

Put

$$
\begin{aligned}
& F=\left\{f \epsilon C(X): f(x)=e^{2 \pi i r / 4} ; r=0,1,2,3 ; x \in X\right\}, \\
& G=\left\{g \epsilon C(Y): g(y)=e^{2 \pi i r / 4} ; r=0,1,2,3 ; y \in \bar{Y}\right\} .
\end{aligned}
$$

Then $S=\{f \otimes g: f \epsilon F, g \in G\}$ is a set of majoration. To show this, let $f^{\prime} \in C(X), g^{\prime} \in C(Y),\left\|f^{\prime}\right\|_{\infty}=\left\|g^{\prime}\right\|_{\infty}=1$. Consider

$$
\left\langle\mu, f^{\prime} \otimes g^{\prime}\right\rangle=\sum_{x, y} \mu(x, y) f^{\prime}(x) g^{\prime}(y)=\varrho e^{i \theta}, \quad \text { say. }
$$

For each $x$, replace $f^{\prime}(x)$ by $f(x)=\exp (2 \pi i r(x) / 4)$, where $r(x)$ $\epsilon\{0,1,2,3\}$ in such a way that

$$
\left|\arg \left\{f(x) \sum_{y} \mu(x, y) g^{\prime}(y)\right\}-\theta\right| \leqslant \pi / 4
$$

It is then clear that

$$
\left|\left\langle\mu, f \otimes g^{\prime}\right\rangle\right| \geqslant \frac{1}{\sqrt{2}} \varrho .
$$

Similarly, replacing $g^{\prime}$ by $g \in G$, we obtain

$$
\|\mu\|_{\mathbb{A}^{*}(E)}=\sup \left|\left\langle\mu, f^{\prime} \otimes g^{\prime}\right\rangle\right| \leqslant 2 \sup _{S}|\langle\mu, f \otimes g\rangle| .
$$

THEOREM 3.2. Let $X, Y$ be compact Hausdorff spaces and let $\left\{S_{n}\right\}_{n=1}^{\infty}$ be a collection of $n$-squares of $X \times Y$. Let $\delta_{x}$ be the Dirac measure of $a$ point $x \in S_{n}$ and let $\Delta_{n}=\left\{\delta_{x}\right\}_{x \in S_{n}}$. Let also $\theta_{n}$ be a random measure equidistributed in $\Delta_{n}$ and put

$$
\xi_{n}=\frac{1}{p_{n}} \sum_{j=1}^{p_{n}} \theta_{n j}
$$

where the $\theta_{n j}$ are $p_{n}$ independent copies of $\theta_{n} . \xi_{n}$ and $\xi_{m}$ are to be independent when $n \neq m$. Put $E_{n}=\operatorname{supp} \xi_{n}$.

Then if $n p_{n}^{-1} \rightarrow 0$ as $n \rightarrow \infty$, the set $H=\bigcup_{n=1}^{\infty} E_{n}$ is almost surely a set of analyticity.

We start by proving two lemmas.

LEMMA 3.3. Let $m$ and $t$ be relatively prime positive integers and put $n=m t$. Let $\mu$ and $\boldsymbol{v}$ denote the Haar measures on $\boldsymbol{Z}(m), \boldsymbol{Z}(n)$ respectively. Then to any real function $f \in A(\boldsymbol{Z}(m))$ there corresponds a real function, $g \in A(\boldsymbol{Z}(n))$ with

$$
\|f\|_{\mathcal{A}}=\|g\|_{\mathcal{A}} \quad \text { and } \quad\left\|\mu e^{i f}\right\|_{\mathcal{A}^{*}}=\left\|\nu e^{i g}\right\|_{\mathcal{A}^{*}} .
$$

Proof. Since $(m, t)=1$, we can write $\boldsymbol{Z}(n)=\boldsymbol{Z}(m) \times \boldsymbol{Z}(t)$ and $\boldsymbol{Z}(n)^{\wedge}=\boldsymbol{Z}(m)^{\wedge} \times \boldsymbol{Z}(t)^{\wedge}$. Let $p$ be the corresponding quotient map $p: \boldsymbol{Z}(n) \rightarrow \boldsymbol{Z}(m)$. Given $f \in A(\boldsymbol{Z}(m))$, we define $g=\check{p} f \in A(\boldsymbol{Z}(n))$ by $g(z)$ $=f(p z)$ for each $z \in \boldsymbol{Z}(n)$. Clearly the map $\check{p}$ is a monomorphism and if $f$ is a real function so is $g$.

Any character $\chi \in \boldsymbol{Z}(n)^{\wedge}$ can be written in the form $\chi=\left(\chi_{m}, \chi_{t}\right)$, where $\chi_{m}, \chi_{t}$ are elements of $\boldsymbol{Z}(m)^{\wedge}, \boldsymbol{Z}(t)^{\wedge}$ respectively. Similarly, we write $z=(x, y) \in \boldsymbol{Z}(n)$, where $x \in \boldsymbol{Z}(m)$ and $y \in \boldsymbol{Z}(t)$. Then we have

$$
\begin{aligned}
\hat{g}(\chi) & =(1 / n) \sum_{\mathbf{Z}(n)} g(z) \bar{\chi}(z) \\
& =(1 / t) \sum_{\mathbf{Z}(t)}\left(1 / m \sum_{\mathbf{Z}(m)} f(x) \bar{\chi}_{m}(x)\right) \bar{\chi}_{t}(y) \\
& =\left\{\begin{array}{cl}
\hat{f}\left(\chi_{m}\right) & \text { if } \chi_{t} \equiv 1, \\
0 & \text { otherwise. }
\end{array}\right.
\end{aligned}
$$

Hence $\|f\|_{A}=\|g\|_{A}$ and similarly, $\left\|\mu e^{i f}\right\|_{A^{*}}=\left\|\nu e^{i g}\right\|_{A^{*}}$.

In the next lemma, $C>0$ and $\alpha>0$ are absolute constants, the values of which are irrelevant for our purposes. 
LEMma 3.4. Let $t$ be a positive integer. We can find a positive integer $n$ such that if $R$ is any integer with $O<R \leqslant t$, then there exists a real function $f \in A(\boldsymbol{Z}(n))$ satisfying

$$
\|f\|_{A}<R \quad \text { and } \quad\left\|\nu e^{i f}\right\|_{A^{*}}<e^{-\alpha R},
$$

where $\boldsymbol{v}$ is the Haar measure on $\boldsymbol{Z}(n)$.

Proof. By [5] there are positive real numbers $k_{1}, k_{2}$ such that if $m>k_{1}$ is a positive integer, there exists a real function $f \in A(\boldsymbol{Z}(m))$ with

$$
\|f\|_{\mathcal{A}}<k_{2} \log m \quad \text { and } \quad\left\|\mu e^{i f}\right\|_{A^{*}}<m^{-1 / 4},
$$

where $\mu$ is the Haar measure on $\boldsymbol{Z}(m)$. Writing $R=\left[k_{2} \log m\right]+1$, we obtain

$$
\|f\|_{A}<R \quad \text { and } \quad\left\|\mu e^{i f}\right\|_{A^{*}}<e^{-\alpha R}
$$

for some $a>0$. It follows that if $R$ is a large enough positive integer, there is an integer $m^{\prime}$ such that for any integer $m$ with $m^{\prime} \leqslant m \leqslant 2 m^{\prime}$, we can find a real function $f \in A(\boldsymbol{Z}(m))$, satisfying (3.5). By Bertrand's postulate (see e.g. [1]), we can find a prime, $m_{R}$ say, such that $m^{\prime} \leqslant m_{R}$ $\leqslant 2 m^{\prime}$. If we put $n=1 . c . m\left\{m_{R}\right\}_{C<R \leqslant t}$, it follows from lemma 3.3 that for any $R$ with $C \leqslant R \leqslant t$, we can find $f \in A(Z(n))$ satisfying the inequalities of lemma 3.4 .

Proof of theorem 3.2. For any positive integer $n$ we identify $S_{n}$ once and for all with $\boldsymbol{Z}(n) \times \boldsymbol{Z}(n)$. The map $M: A(\boldsymbol{Z}(n)) \rightarrow V\left(S_{n}\right)$ of lemma 2.1 identifies $A(\boldsymbol{Z}(n))$ isometrically with a closed subalgebra, $A^{\prime}\left(S_{n}\right)$, of $V\left(S_{n}\right) \cdot A^{\prime}\left(S_{n}\right)$ consists of those functions $g$ of $V\left(S_{n}\right)$ which satisfy $g(x, y)=g(z, t)$ whenever $x+y=z+t$ ([11], 8.1).

Let $t$ be a positive integer. By lemma 3.4 and the remarks above, there is a positive integer $n=n_{t}$ such that for any integer $R$ with $C<R \leqslant t$ there is a real function $f=f_{R, t} \in A^{\prime}\left(S_{n}\right)$ such that

$$
\|f\|_{\nu\left(S_{n}\right)}<R \quad \text { and } \quad\left\|e^{i f} v_{n}\right\|_{A^{\prime}\left(S_{n}\right)^{*}}<e^{-a R}
$$

where $v_{n}$ is the equidistributed positive measure of total mass 1 on $S_{n}$, and $C$ and $\alpha$ are the constants of lemma 3.4

Moreover,

$$
\left\|e^{i f} v_{n}\right\|_{\nabla^{*}\left(S_{n}\right)}<e^{-\alpha R}
$$

for if not, there exists $g \in V\left(S_{n}\right)$ with

Consider

$$
\|g\|_{V} \leqslant 1 \quad \text { and } \quad\left|\left\langle g, e^{i f} v_{n}\right\rangle\right| \geqslant e^{-a R} .
$$

$$
\tilde{g}=\frac{1}{n} \sum_{x \in \boldsymbol{Z}(n)} g_{x},
$$

where $g_{x}$ is defined by

$$
g_{x}(y, z)=g(y+x, z-x)
$$

for all $y, z \in \boldsymbol{Z}(n)$. Clearly $\tilde{g} \in A^{\prime}\left(S_{n}\right)$,

$$
\|\tilde{g}\|_{v} \leqslant 1 \quad \text { and } \quad\left|\left\langle\tilde{g}, e^{i f} v_{n}\right\rangle\right| \geqslant e^{-\alpha R},
$$

which is a contradiction. So we have a real function $f \in V\left(S_{n}\right)$ with

$$
\|f\|_{\nu}<R \quad \text { and } \quad\left\|e^{i f} v_{n}\right\|_{\nu^{*}}<e^{-a R} \text {. }
$$

Let $\xi_{n}$ be the random measure of the statement of the theorem. Let $\left\{k_{1}, \ldots, k_{s}\right\}$ be a set of majoration of \|\|$_{r\left(S_{n}\right)}$ with respect to \|\|$_{\infty}$ where $s$ is the arithmetic diameter of $S_{n}$. Then we have

$$
\left\|e^{i f} \xi_{n}\right\|_{r^{*}} \leqslant 2 \sup _{r=1, \ldots, s}\left|\left\langle k_{r}, e^{i f} \xi_{n}\right\rangle\right|
$$

Let $Z^{r}$ be the random variable defined by

$$
Z^{r}(x, y)=k_{r}(x, y) \exp (i f(x, y))
$$

as $(x, y)$ is chosen at random in $S_{n}$. Then we have

$$
\left|\mathscr{E}\left(Z^{r}\right)\right| \leqslant\left\|e^{i f} v_{n}\right\|_{\Gamma^{*}}<e^{-\alpha R}
$$

and

$$
\left\langle k_{r}, e^{i f} \xi_{n}\right\rangle=\frac{1}{p_{n}} \sum_{q=1}^{p_{n}} Z_{q}^{r},
$$

where $Z_{q}^{r}$ is a copy of $Z^{r}$. We shall use the following lemma (for a proof see [9]):

LEMMA 3.7. Let $Z$ be a complex-valued random variable with $|Z| \leqslant 1$ and $\mathscr{E}(Z)=\alpha$. Let $Z_{1}, \ldots, Z_{p}$ be $p$ independent copies of $Z$ and let

$$
Z^{*}=p^{-1}\left(Z_{1}+\ldots+Z_{p}\right) \text {. }
$$

Then, for any $\varepsilon>0$,

$$
P\left\{\left|Z^{*}-\alpha\right|>\varepsilon\right\}<4 e^{-\beta p \varepsilon^{2}}
$$

for some $\beta>0$.

From this, and the inequalities above, we obtain

$P\left\{\left|\left\langle k_{r}, e^{i f} \xi_{n}\right\rangle-\mathscr{E}\left(Z^{r}\right)\right|>\varepsilon\right.$ for some $\left.r \in[1,2, \ldots s]\right\}<4 s e^{-\beta p_{n} \varepsilon^{2}}$

$$
\leqslant 4^{2 n+1} e^{-\beta p_{n} \varepsilon^{2}} \text {. }
$$

Hence

$$
P\left\{\left\|e^{i f} \xi_{n}\right\|_{\nabla^{*}}>2 e^{-a R}+2 \varepsilon\right\} \leqslant \exp \left(k(2 n+1)-\beta p_{n} \varepsilon^{2}\right)
$$


Writing $f_{n}$ for $\left.f\right|_{E_{n}}$, we have

$$
\left\langle e^{i f} \xi_{n}, e^{-i f_{n}}\right\rangle=1
$$

and, extending $f_{n}$ to a real function $g \in V(H)$ such that $\|g\|_{V(H)}<2 R$, we get

$$
P\left\{\left\|e^{i \theta}\right\|_{\Gamma(H)}<\left(2 e^{-\alpha R}+2 \varepsilon\right)^{-1}\right\}<\exp \left(k(2 n+1)-\beta p_{n} \varepsilon^{2}\right) .
$$

We now choose positive numbers $\varepsilon_{n}$ such that $\varepsilon_{n} \rightarrow 0$ and $n / p_{n} \varepsilon_{n} \rightarrow 0$ as $n \rightarrow \infty$ and we consider the functions $g_{R, t} \in V(H)$ corresponding to $f_{R, t} \in V\left(S_{n_{t}}\right)$. Since

$$
P\left\{\| e^{i g_{R, t} \|_{\Gamma}}<\left(2 e^{-a R}+2 \varepsilon_{n_{t}}\right)^{-1}\right\}<\exp \left(k\left(2 n_{t}+1\right)-\beta p_{n_{t}} \varepsilon_{n_{t}}^{2}\right),
$$

it is clear that, given $\delta>0$, we can find, for each $R>C$, an integer $t_{R}$ such that

$$
P\left\{\left\|e^{i g_{R}}\right\|_{V} \leqslant \frac{1}{3} e^{a R}\right\}<\delta / 2^{R}
$$

(where $g_{R}=g_{R, t_{R}} \in V(H)$ ). But $\left\|g_{R}\right\|_{V}<2 R$, so we can deduce (by the criterion of [3]) that

$$
P\{H \text { is not a set of analyticity }\}<\delta .
$$

Hence $H$ is almost surely a set of analyticity.

4. For some particular subsets of $X \times Y$ we can get direct transpositions of the results of Malliavin and Katznelson [5], [6].

Definition 4.1. Let $X, Y$ be sets and let $S_{n}=X_{n} \times Y_{n}$ be an $n$-square in $X \times Y$. If $G$ is an abelian group of order $n$ and $X_{n}, Y_{n}$ are identified with $G$, the $G$-fibres of $S_{n}$ are the equivalence classes of points of $S_{n}$ corresponding to the relation

$$
(x, y) \sim(z, t) \Leftrightarrow x+y=z+t .
$$

A subset $E$ of $S_{n}$ is called a $G$-diagonal subset if

$$
(x, y) \in E \Leftrightarrow(z, t) \epsilon E
$$

for all $x, y, z, t \in G$ having $x+y=z+t$. (These definitions are, of course, dependent on the particular identifications of $X_{n}, Y_{n}$ with G.)

TheOREM 4.2. Let $X, Y$ be compact Hausdorff spaces and let $H$ be a closed subset of $X \times Y$. Suppose $H$ contains a set of the form $\bigcup_{n=1}^{\infty} E_{n}$, where

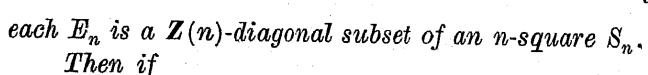

$$
\varlimsup_{n \rightarrow \infty}\left|E_{n}\right|>n^{1+\varepsilon}
$$

for some $\varepsilon>0, H$ is a set of analyticity.
Proof. Let $\boldsymbol{F}_{n}=\left\{z \in \boldsymbol{Z}(n): z=x+y\right.$ for some $\left.(x, y) \in E_{n}\right\}$. Clearly $\left|F_{n}\right|=\left|E_{n}\right| / n$ and thus

$$
\varlimsup_{n \rightarrow \infty}\left|F_{n}\right|>n^{\varepsilon} .
$$

It then follows from [5], that, given $R>0$, we can find a real function $f \in A\left(F_{n}\right)$ for some $n$, having

$$
\|f\|_{A\left(F_{n}\right)}<R \quad \text { and } \quad\left\|e^{i f}\right\|_{A\left(F_{n}\right)}>e^{a R}
$$

for some positive constant $a$. Applying the mapping $M: A(\boldsymbol{Z}(n)) \rightarrow V\left(S_{n}\right)$ we obtain the required result.

THEOREM 4.3. Let $X, Y$ be compact Hausdorff spaces and let $H$ be $a$ closed subset of $X \times Y$. Let $H$ contain a set of the form $\bigcup_{n=1}^{\infty} E_{n}$, where $E_{n}$ is the union of $\left|E_{n}\right| / n \boldsymbol{Z}(n)$-fibres of $S_{n}$ chosen at random.

Then if

$$
(n \log n)\left|E_{n}\right|^{-1} \rightarrow 0
$$

as $n \rightarrow \infty, H$ is almost surely a set of analyticity.

Proof. As above, using [6], Theorem 1, in place of [5].

\section{References}

[1] G. H. Hardy and E. M. Wright, An introduction to the theory of numbers, IV ed., p. 343.

[2] H. Helson, J.-P. Kahane, Y. Katznelson and W. Rudin, The functions which operate on Fourier transforms, Acta Math. 102 (1959), p. 135-157.

[3] J.-P. Kahane and Y. Katznelson, Contribution à deux problèmes concernant les fonetions de la classe A, Isr. J. Math. 1 (1963), p. 110-131.

[4] Y. Katznelson, Calcul symbolique dans les algèbres homogènes, C. R. Acad. Sc. Paris 254 (1962), p. 2700-2702.

[5] - and P. Malliavin, Un critère d'analyticité pour les algèbres de restriction, ibidem 261 (1965), p. 4964-4967.

[6] - Vérification statistique de la conjecture de la dichotomie sur une classe d'algèbres de restriction, ibidem 262 (1966), p. 490-492.

[7] W. Rudin, Fourier analysis on groups, Interscience, 1962, p. 166 et seq.

[8] T. Kövari, V. T. Sós and P. Turàn, On a problem of $K$. Zarantiewicz, Coll. Math. 3 (1954,) p. 50-57.

[9] T. V. Uspensky, Introduction to mathematical probability, 1937, p. 204-205.

[10] N. Th. Varopoulos, Lecture notes at the Summer School on Topological Algebra Theory, Bruges, September 1966.

[11] - Tensor algebras and harmonic analysis, Acta Math. 119 (1967,) p. 51-112. 